\title{
Evolution of Genome Organization by Duplication and Loss: an Alignment Approach
}

\author{
Patrick Holloway $^{1}$, Krister Swenson $^{2}$, David Ardell ${ }^{3}$, and Nadia El-Mabrouk ${ }^{4}$ \\ 1 Département d'Informatique et de Recherche Opérationnelle (DIRO), Université de Montréal, H3C 3J7, \\ Canada, patrick.holloway@umontreal.ca \\ 2 DIRO and University of McGill Computer Science, swensonk@iro.umontreal.ca \\ 3 Center for Computational Biology, School of Natural Sciences, 5200 North Lake Road, University of \\ California, Merced, CA 95343, dardell@ucmerced.edu \\ 4 DIRO, mabrouk@iro.umontreal.ca
}

Keywords: Comparative Genomics, Gene order, Duplication, Loss, Linear Programming, Alignment, Bacillus, tRNA.

\begin{abstract}
We present a comparative genomics approach for inferring ancestral genome organization and evolutionary scenarios, based on a model accounting for content-modifying operations. More precisely, we focus on comparing two ordered gene sequences with duplicated genes that have evolved from a common ancestor through duplications and losses; our model can be grouped in the class of "Block Edit" models. From a combinatorial point of view, the main consequence is the possibility of formulating the problem as an alignment problem. On the other hand, in contrast to symmetrical metrics such as the inversion distance, duplications and losses are asymmetrical operations that are applicable to one of the two aligned sequences. Consequently, an ancestral genome can directly be inferred from a duplication-loss scenario attached to a given alignment. Although alignments are a priori simpler to handle than rearrangements, we show that a direct approach based on dynamic programming leads, at best, to an efficient heuristic. We present an exact pseudo-boolean linear programming algorithm to search for the optimal alignment along with an optimal scenario of duplications and losses. Although exponential in the worst case, we show low running times on real datasets as well as synthetic data. We apply our algorithm in a phylogenetic context to the evolution of stable RNA (tRNA and rRNA) gene content and organization in Bacillus genomes. Our results lead to various biological insights, such as rates of ribosomal RNA proliferation among lineages, their role in altering tRNA gene content, and evidence of tRNA class conversion.
\end{abstract}

List of Topics: Molecular Evolution, Genomics. 


\section{Introduction}

During evolution, genomes continually accumulate mutations. In addition to base mutations and short insertions or deletions, genome-scale changes affect the overall gene content and organization of a genome. Evidence of these latter kinds of changes are observed by comparing the completely sequenced and annotated genomes of related species. Genome-scale changes can be subdivided into two categories: (1) the rearrangement operations that shuffle gene orders (inversions, transpositions, and translocations), and (2) the content-modifying operations that affect the number of gene copies (gene insertions, losses, and duplications). In particular, gene duplication is a fundamental process in the evolution of species [25], especially in eukaryotes [5, 9, 12, 16, 22, 34], where it is believed to play a leading role for the creation of novel gene function. In parallel, gene losses through pseudogenization and segmental deletions, appear generally to maintain a minimum number of functional gene copies $[5,9,10,12,16,22,25]$. Transfer RNAs (tRNAs) are typical examples of gene families that are continually duplicated and lost $[3,28,31,35]$. Indeed, tRNA clusters (or operons in microbial genomes) are highly dynamic and unstable genomic regions. In Escherichia coli for example, the rate of tRNA gene duplication/loss events has been estimated to be about one event every 1.5 million years $[3,35]$.

One of the main goals of comparative genomics is to infer evolutionary histories of gene families, based on the comparison of the genomic organization of extant species. Having an evolutionary perspective of gene families is a key step towards answering many fundamental biological questions. For example, tRNAs are essential to establishing a direct link between codons and their translation into amino-acids. Understanding how the content and organization of tRNAs evolve is essential to the understanding of the translational machinery, and in particular, the variation in codon usage among species $[20,11]$.

In the genome rearrangement approach to comparative genomics, a genome is modeled as one or many (in case of many chromosomes) linear or circular sequences of genes (or other building blocks of a genome). When each gene is present exactly once in a genome, sequences can be represented as permutations. In the most realistic version of the rearrangement problem, a sign (+ or -) is associated with a gene, representing its transcriptional orientation. Most genome rearrangement studies have focused on signed permutations. The pioneering work of Hannenhalli and Pevzner in $1995[17,18]$, has led to efficient algorithms for computing the inversion and/or translocation distance between two signed permutations. Since then, many other algorithms have been developed to compare permutations subject to various rearrangement operations and based on different distance measures. These algorithms have then been used from a phylogenetic perspective to infer ancestral permutations $[24,6,8,23,30]$ and evolutionary scenarios on a species tree. An extra degree of difficulty is introduced in the case of sequences containing multiple copies of the same gene, as the one-to-one correspondence between copies is not established in advance. A review of the methods used for comparing two ordered gene sequences with duplicates can be found in $[14,15]$. They can be grouped into two main classes. The "Match-and-Prune" model aims at transforming strings into permutations, so as to minimize a rearrangement distance between the resulting permutations. On the other hand, the "Block Edit" model consists of performing the minimum number of "allowed" rearrangement and content-modifying operations required to transform one string into the other. Most studied distances and ancestral inference problems in this category are NP-complete [15].

In this paper, we focus on comparing two ordered gene sequences with duplicates that have evolved from a common ancestor through duplications and losses. In contrast to the approaches cited above and reviewed in [15], only content-modifying operations are considered. Such a simplified model is required to study the evolution of gene families mainly affected by duplications and losses, for which a general model involving rearrangement events may be misleading. From a combinatorial point of view, the main consequence of removing rearrangement operations is the fact that gene organization is preserved, which allows us to reformulate the problem of comparing two gene orders as an alignment problem. On the other hand, in contrast to symmetrical metrics such 
as the Hamming distance for nucleotide sequences, or the inversion distance for ordered sequences, duplication and loss are asymmetrical operations that are applicable to one of the two aligned sequences. Consequently an ancestral genome can directly be inferred from a duplication-loss scenario attached to a given alignment.

Although alignments are a priori simpler to handle than rearrangements, there is no direct way of inferring optimal alignments together with a related duplication-loss scenario for two gene orders, as detailed in Section 4. Even our simpler goal of finding an alignment is fraught with difficulty as a naive branch-and-bound approach to compute such an alignment is non-trivial; trying all possible alignments with all possible duplication and loss scenarios for each alignment is hardly practicable. As it is not even clear how, given an alignment, we can assign duplications and losses in a parsimonious manner, we present in Section 4.1 a pseudo-boolean linear programming (PBLP) approach to search for the optimal alignment along with an optimal scenario of duplications and losses. The disadvantage of the approach is that, in the worst case, an exponential number of steps could be used by our algorithm. On the other hand, we show in Section 5.2 that for real data, and larger simulated genomes, the running times are quite reasonable. Further, the PBLP is flexible in that a multitude of weighting schemes for losses and duplications could be employed to, for example, favor certain duplications over others, or allow for gene conversion. In Section 5.1, we apply our algorithm in a phylogenetic context to infer the evolution of stable RNA (tRNA and rRNA) gene content and organization in various genomes from the genus Bacillus, a so-called "low G+C" gram-positive clade of Firmicutes that includes the model bacterium B. subtilis as well as the agent of anthrax. Stable RNA operon organization in this group is interesting because it has relatively fewer operons that are much larger and contain more segmental duplicates than other bacterial groups. We obtained results leading to various biological insights, such as more accurate quantification of ribosomal RNA operon proliferation, their role in altering tRNA gene content, and evidence of tRNA gene class conversion.

\section{Research context}

The evolution of $g$ genomes is often represented by a phylogenetic (or species) tree $T$, binary or not, with exactly $g$ leaves, each representing a different genome. When such a species tree $T$ is known for a set of species, then we can use the gene order information of the present-day genomes to infer gene order information of ancestral genomes identified with each of the internal nodes of the tree. This problem is known in the literature as the "small" phylogeny problem, in contrast to the "large" phylogeny problem which is one of finding the actual phylogenetic tree $T$.

Although our methods may be extended to arbitrary genomes, we consider single chromosomal (circular or linear) genomes, represented as gene orders with duplicates. More precisely, given an alphabet $\Sigma$ where each character represents a specific gene family, a genome or string is a sequence of characters from $\Sigma$ where each character may appear many times. As the content-modifying operations considered in this paper do not change gene orientation, we can assume, w.l.o.g. that genes are unsigned. For example, given $\Sigma=\{a, b, c, d, e\}, A=$ "ababcd" is a genome containing two gene copies from the gene family identified by $a$, two genes from the gene family $b$, and a single gene from each family $c$ and $d$. A gene in a genome $A$ is a singleton if it appears exactly once in $A$ (for example $c$ and $d$ in $A$ ), and a duplicate otherwise ( $a$ and $b$ in $A$ above).

Let $\mathcal{O}$ be a set of "allowed" evolutionary operations. The set $\mathcal{O}$ may include organizational operations such as Reversals (R) and Transpositions (T), and content-modifying operations such as Duplications (D), Losses (L) or Insertions (I). For example, $\mathcal{O}=\{R, D, L\}$ is the set of operations in an evolutionary model involving reversals, duplications and losses. In the next section, we will formally define the operations involved in our model of evolution.

Given a genome $A$, a mutation on $A$ is characterized by an operation $O$ from $\mathcal{O}$, the substring of $A$ that is affected by the mutation, as well as possibly other characteristics such as the position of 
the re-inserted, removed (in case of transposition), or duplicated substring. For simplicity, consider a mutation $O(k)$ to be characterized solely by the operation $O$ from $\mathcal{O}$, and the size $k$ of the substring affected by the mutation. Consider $c(O(k))$ to be a cost function defined on mutations. Finally, given two genomes $A$ and $X$, an evolutionary history $O_{A \rightarrow X}$ from $A$ to $X$ is a sequence of mutations (possible of length 0) transforming $A$ into $X$.

Let $A, X$ be two strings on $\Sigma$ with $A$ being a potential ancestor of $X$, meaning that there is at least one evolutionary history $O_{A \rightarrow X}=\left\{O_{1}\left(k_{1}\right), \cdots, O_{l}\left(k_{l}\right)\right\}$ from $A$ to $X$. Then the cost of $O_{A \rightarrow X}$ is:

$$
C\left(O_{A \rightarrow X}\right)=\sum_{i=1}^{l} c\left(O_{i}\left(k_{i}\right)\right)
$$

Now let $\mathcal{O}_{A \rightarrow X}$ be the set of possible histories transforming $A$ into $X$. Then we define:

$$
C(A \rightarrow X)=\min _{O_{A \rightarrow X} \in \mathcal{O}_{A \rightarrow X}} C\left(O_{A \rightarrow X}\right)
$$

Then, the small phylogeny problem can be formulated as one of finding strings at internal nodes of a given tree $T$ that minimize the total cost:

$$
C(T)=\sum_{\text {all branches } b_{i} \text { of } T} C\left(X_{i, 1} \rightarrow X_{i, 2}\right)
$$

where $X_{i, 1}, X_{i, 2}$ are the strings labeling the nodes of $T$ adjacent to the branch $b_{i}$, with the node labeled $X_{i, 1}$ being the parent of the node labeled $X_{i, 2}$.

For most restrictions on genome structure and models of evolution, the simplest version of the small phylogeny problem - the median of three genomes - is NP-hard [7, 26,32]. When duplicate genes are present in the genomes, even finding minimum distances between two genomes is almost always an NP-Hard task [19]. In this paper, we focus on cherries of a species tree (i.e. on subtrees with two leaves). The optimization problem we consider can be formulated as follows:

\section{Two Species Small Phylogeny Problem:}

InPuT: Two genomes $X$ and $Y$.

Output: A potential common ancestor $A$ of $X$ and $Y$ minimizing the cost

$$
C(A \rightarrow X)+C(A \rightarrow Y) .
$$

Solving the Two Species Small Phylogeny Problem (2-SPP) can be seen as a first step towards solving the problem on a given phylogenetic tree $T$. The most natural heuristic to the Small Phylogeny Problem, that we will call the SPP-HEuRIstic, is to traverse $T$ depth-first, and to compute successive ancestors of pairs of nodes. Such a heuristic can be used as the initialization step of the steinerization method for SPP [30,4]. The sets of all optimal solutions output by an algorithm for the 2-SPP applied to all pairs of nodes of $T$ (in a depth-first traversal) can alternatively be used in an iterative local optimization method, such as the dynamic programming method developed in $[21]$.

\section{The Duplication and Loss Model of Evolution}

Our evolutionary model accounts for two operations, Duplication (denoted $D$ ) and Loss (denoted $L)$. In other words $\mathcal{O}=\{D, L\}$, where $D$ and $L$ are defined as follows. Let $X[i \ldots i+k]$ denote the substring $X_{i} X_{i+1} \cdots X_{i+k}$ of $X$. 
- D: A Duplication of size $k+1$ on $X=X_{1} \cdots X_{i} \cdots X_{i+k} \cdots X_{j} X_{j+1} \cdots X_{n}$ is an operation that copies the substring $X[i \ldots i+k]$ to a location $j$ of $X$ outside the interval $[i, i+k]$ (i.e. preceding $i$ or following $i+k$ ). In the latter case, $D$ transforms $X$ into

$$
X^{\prime}=X_{1} \cdots \underline{X_{i} \cdots X_{i+k}} \cdots X_{j-1} \underline{X_{i} \cdots X_{i+k}} X_{j+1} \cdots X_{n}
$$

We call the original copy $X[i \ldots i+k]$ the origin, and the copied string the product of the duplication $D$.

- L: A Loss of size $k$ is an operation that removes a substring of size $k$ from $X$.

Notice that gene insertions could be considered in our model as well. In particular, our linear programming solution is applicable to an evolutionary model involving insertions, in addition to duplications and losses. We ignore insertions for two main reasons: (1) insertions and losses are two symmetrical operations that can be interchanged in an evolutionary scenario. Distinguishing between insertions and losses may be possible on a phylogeny, but cannot be done by comparing two genomes; (2) gene insertions are usually due to lateral gene transfer, which may be rare events compared to nucleotide-level mutations that eventually transform a gene into a pseudogene.

As duplication and loss are content-modifying operations that do not shuffle gene order, the Two Species Small Phylogeny Problem can be posed as an alignment problem. However, the only operations that are "visible" on an alignment are the events on an evolutionary history that are not obscured by subsequent events. Moreover, as duplications and losses are asymmetrical operations, an alignment of two genomes $X$ and $Y$ does not reflect an evolutionary path from $X$ to $Y$ (as operations going back to a common ancestor are not defined), but rather two paths going from a common ancestor to both $X$ and $Y$. A precise definition follows.

Definition 1. Let $X$ and $Y$ be two genomes. $A$ visible history of $X$ and $Y$ is a triplet $\left(A, O_{A \rightarrow X}\right.$, $O_{A \rightarrow Y}$ ) where $A$ is a potential ancestor of both $X$ and $Y$, and $O_{A \rightarrow X}$ (respectively $O_{A \rightarrow Y}$ ) are evolutionary histories from $A$ to $X$ (respectively from $A$ to $Y$ ) verifying the following property: Let $D$ be a duplication in $O_{A \rightarrow X}$ or $O_{A \rightarrow Y}$ copying a substring $S$. Let $S_{1}$ be the origin and $S_{2}$ be the product of $D$. Then $D$ is not followed by any other operation inserting (by duplication) genes inside $S_{1}$ or $S_{2}$, or removing (by loss) genes from $S_{1}$ or $S_{2}$. We call a visible ancestor of $X$ and $Y$ a genome $A$ belonging to a visible history $\left(A, O_{A \rightarrow X}, O_{A \rightarrow Y}\right)$ of $X$ and $Y$.

We now define an alignment of two genomes.

Definition 2. Let $X$ be a string on $\Sigma$, and let $\Sigma^{-}$be the alphabet $\Sigma$ augmented with an additional character "-". An extension of $A$ is a string $A^{-}$on $\Sigma^{-}$such that removing all occurrences of the character " - "from $A^{-}$leads to the string A.

Definition 3. Let $X$ and $Y$ be two strings on $\Sigma$. An alignment of size $\alpha$ of $X$ and $Y$ is a pair ( $\left.X^{-}, Y^{-}\right)$extending $(X, Y)$ such that $\left|X^{-}\right|=\left|Y^{-}\right|=\alpha$, and for each $i, 1 \leq i \leq \alpha$, the two following properties hold:

- If $X_{i}^{-} \neq "$-" and $Y_{i}^{-} \neq$" - " then $X_{i}^{-}=Y_{i}^{-}$;

- $X_{i}^{-}$and $Y_{i}^{-}$cannot be both equal to "-".

Let $\mathcal{A}=\left(X^{-}, Y^{-}\right)$be an alignment of $X$ and $Y$ of size $\alpha$. It can be seen as a $2 \times \alpha$ matrix, where the $i$ th column $\mathcal{A}_{i}$ of the alignment is just the $i$ th column of the matrix. A column is a match iff it does not contain the character '-', and a gap otherwise. A gap $\left[\begin{array}{c}X_{i} \\ -\end{array}\right]$ is either part of a loss in $Y$, or part of a duplication in $X$ (only possible if the character $X_{i}$ is a duplicate in $X$ ). The same holds for the column $\left[\begin{array}{c}- \\ Y_{j}\end{array}\right]$. An interpretation of $\mathcal{A}$ as a sequence of duplications and losses is called a labeling of $\mathcal{A}$. The cost of a labeled alignment is the sum of costs of all underlying operations. 
As duplications and losses are asymmetric operations that are applied explicitly to one of the two strings, each labeled alignment $\mathcal{A}$ of $X$ and $Y$ leads to a unique common ancestor $A$ for $X$ and $Y$. The following theorem (whose proof is left to the appendix) shows that this, and the converse is true.

Theorem 1. Given two genomes $X$ and $Y$, there is a one-to-one correspondence between labeled alignments of $X$ and $Y$ and visible ancestors of $X$ and $Y$.

See Figure 1 for an example.
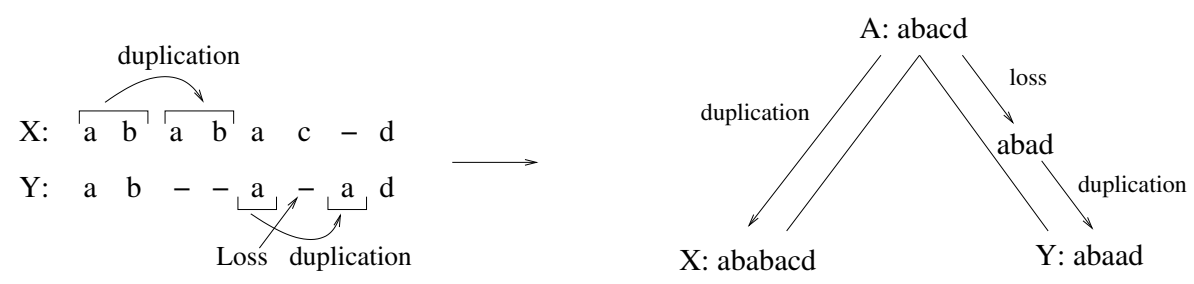

Fig. 1: Left: a labeled alignment between two strings $X=$ ababacd and $Y=$ abaad. Right: the ancestor $A$ and two histories respectively from $A$ to $X$ and from $A$ to $Y$ obtained from this alignment. The order of operations in the history from $A$ to $Y$ is arbitrary.

In other words, Theorem 1 states that the Two Species Small Phylogeny Problem reduces in the case of the Duplication-Loss model of evolution to the following optimization problem.

\section{Duplication-Loss Alignment Problem:}

InPUT: Two genomes $X$ and $Y$ on $\Sigma$.

Output: A labeled alignment of $X$ and $Y$ of minimum cost.

\section{Method}

Although alignments are a priori simpler to handle than rearrangements, a straightforward way to solve the Duplication-Loss Alignment Problem is not known. We show in the following paragraphs, that a direct approach based on dynamic programming leads, at best, to an efficient heuristic, with no guarantee of optimality.

Let $X$ be a genome of size $n$ and $Y$ be a genome of size $m$. Denote by $X[1 \ldots i]$ the prefix of size $i$ of $X$, and by $Y[1 \ldots j]$ the prefix of size $j$ of $Y$. Let $C(i, j)$ be the minimum cost of a labeled alignment of $X[1 \ldots i]$ and $Y[1 \ldots j]$. Then the problem is to compute $C(m, n)$.

DP: A natural idea would be to consider a dynamic programming approach (DP), computing $C(i, j)$, for all $1 \leq i \leq n$ and all $1 \leq j \leq m$. Consider the variables $M(i, j), D_{X}(i, j), D_{Y}(i, j)$, $L_{X}(i, j)$ and $L_{Y}(i, j)$ which reflect the minimum cost of an alignment $\mathcal{A}_{i, j}$ of $X[1 \ldots i]$ and $Y[1 \ldots j]$ satisfying respectively, the constraint that the last column of $\mathcal{A}_{i, j}$ is a match, a duplication in $X$, a duplication in $Y$, a loss in $X$, or a loss in $Y$. Consider the following recursive formulae.

$-M(i, j)= \begin{cases}C(i-1, j-1) & \text { if } X[i]=Y[j] \\ +\infty & \text { otherwise }\end{cases}$

- $L_{X}(i, j)=\min _{0 \leq k \leq i-1}[C(k, j)+c(L(i-k))]$

(the corresponding formula holds for $L_{Y}(i, j)$ )

- $D_{X}(i, j)= \begin{cases}+\infty & \text { if } X[i] \text { is a singleton } \\ \min _{l \leq k \leq i-1}[C(k, j)+c(D(i-k))] \text { otherwise, }\end{cases}$ where $X[l \ldots i]$ is the longest suffix of $X[1 \ldots i]$ that is a duplication (the corresponding formula holds for $D_{Y}(i, j)$ ). 
The recursions for $D_{X}$ and $D_{Y}$ imply that duplicated segments are always inserted to the right of the origin. Unfortunately, such an assumption cannot be made while maintaining optimality of the alignment. For example, given the cost $c(D(k))=1$ and $c(L(k))=k$, the optimal labeled alignment of $S_{1}=a b x a b x a b$ and $S_{2}=x a b x$ aligns $a b$ of $S_{2}$ with the second $a b$ of $S_{1}$, leading to an optimal history with two duplications inserting the second $a b$ of $S_{1}$ to its left and to its right. Such an optimal scenario cannot be recovered by DP.

DP-2WAY: As a consequence of the last paragraph, consider the two-way dynamic programming approach DP-2WAY that computes $D_{X}(i, j)$ (resp. $D_{Y}(i, j)$ ) by looking for the longest suffix of $X[1 \ldots i]$ (resp. $Y[1 \ldots j]$ ) that is a duplication in the whole genome $X$ (resp. $Y$ ). Unfortunately, DP-2WAY may lead to invalid cyclic evolutionary scenarios, as the same scenario may involve two duplications: one with origin $S_{1}$ and product $S_{2}$, and one with origin $S_{2}$ and product $S_{1}$, where $S_{1}$ and $S_{2}$ are two duplicated strings. This is described in more detail in Section 4.1.

DP-2WAY-UNLABELED: The problem mentioned above with the output of DP-2WAY is not necessarily the alignment itself, but rather the label of the alignment. As a consequence, one may think about a method, DP-2WAY-UNLABELED, that would consider the unlabeled alignment output by DP-2WAY, and label it in an optimal way (e.g. find an evolutionary scenario of minimum cost that is in agreement with the alignment). Notice first that the problem of finding a most parsimonious labeling of a given alignment, is not a priori an easy problem, and there is no direct and simple way to do it. Moreover, although DP-2WAY-UNLABELED is likely to be a good heuristic algorithm to the Duplication-Loss Alignment Problem, it would not be an exact algorithm, as an optimal cyclic alignment is not guaranteed to have a valid labeling leading to an optimal labeled alignment. Figure 2 shows such an example; an optimal cyclic duplication and loss scenario can be achieved by both alignments ( 5 operations), while the optimal acyclic scenario can only be achieved by the alignment of Figure $2 \mathrm{~b}$.

\subsection{The Pseudo-Boolean Linear Program}

Consider genome $X$ of length $n$ and genome $Y$ of length $m$. We show how to compute a labeled alignment of $X$ and $Y$ by use of pseudo-boolean linear programming (PBLP). The alignment that we compute is guaranteed to be optimal. While in the worst case our program could take an exponential number (in the length of the strings) of steps to find the alignment, our formulation has a cubic number of equations variables, and is far more efficient than scoring all possible alignments along with all possible duplication/loss scenarios. We show that practical running times can be achieved on real data in Section 5.2.

For any alignment, an element of the string $X$ could be considered a loss (this corresponds to gaps in the alignment), a match with an element of $Y$, or a duplication from another element in $X$ (these also appear as gaps in the alignment). Thus, in a feasible solution, every element must be "covered" by one of those three possibilities. The same holds for elements of $Y$. Figure 2 shows two possible alignments for a given pair of strings, along with the corresponding set of duplications and losses. In the alignment of Figure $2 \mathrm{a}$, character $\mathrm{x}_{8}$ is covered by the duplication of $\mathrm{x}_{12}$, character $\mathrm{a}_{11}$ is covered by a loss, and character $\mathrm{x}_{5}$ is covered by a match with character $\mathrm{x}_{4}$ in $Y$.

Let $M_{j}^{i}$ signify the match of character $X_{i}$ to character $Y_{j}$ in the alignment. Say character $X_{i}$ could be covered by matches $M_{1}^{i}, M_{2}^{i}, \ldots, M_{p_{i}}^{i}$ or by duplications $D X_{1}^{i}, D X_{2}^{i}, \ldots, D X_{s_{i}}^{i}$. If we consider each of those to be a binary variable (can take value 0 or 1 ) and take the binary variable $L X^{i}$ as corresponding to the possibility that $X_{i}$ is a loss, then we have the following equation to ensure that character $X_{i}$ is covered by exactly one operation:

$$
L X^{i}+M_{1}^{i}+M_{2}^{i}+\cdots+M_{p_{i}}^{i}+D X_{1}^{i}+D X_{2}^{i}+\cdots+D X_{s_{i}}^{i}=1,
$$

where $p_{i}$ and $s_{i}$ are the number of matches and duplications that could cover character $X_{i}$. A potential duplication in $X\left(D X_{l}^{i}\right.$ for some $\left.l\right)$ corresponds to a pair of distinct, but identical, substrings 


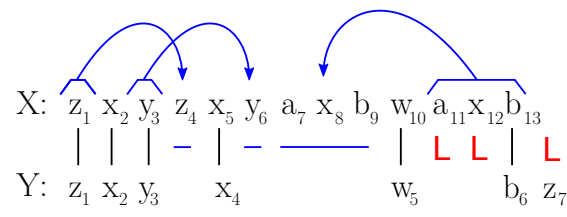

(a) 3 duplications and 3 losses

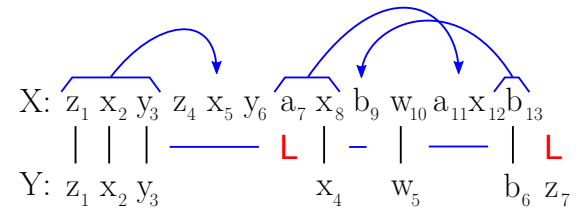

(b) 3 duplications and 2 loss

Fig. 2: Alignments for strings $X=$ "zxyzxyaxbwaxb" and $Y=$ "zxyxwb". We consider the following cost: $c(D(k))=1$ and $c(L(k))=k$ for any integer $k$. Matches are denoted by a vertical bar, losses denoted by an "L", and duplications denoted by bars, brackets, and arrows. Alignment (a) yields 6 operations and implies ancestral sequence "zxyxwaxbz", while (b) yields 5 operations and implies ancestral sequence "zxyaxwbz".

in $X$. Each pair of such substrings yields two potential duplications (substring $A$ was duplicated from substring $B$ or substring $B$ was duplicated from substring $A$ ). Each of the possible $O\left(n^{3}\right)$ duplications gets a variable. Each position in $Y$ gets a similar equation to Equation 1.

The order of the matches with respect to the order of the strings must be enforced. For example, in Figure 2 it is impossible to simultaneously match $\mathrm{w}_{10}$ and $\mathrm{x}_{12}$ from $X$ with $\mathrm{w}_{5}$ and $\mathrm{x}_{4}$ of string $Y$; the assignment of variables corresponding to this case must be forbidden in the program. Thus, we introduce equations enforcing the order of the matches. Recall that $M_{j}^{i}$ is the variable corresponding to the match of the $i$ th character from $X$ with the $j$ th character from $Y$. The existence of match $M_{j}^{i}$ implies that any match $M_{l}^{k}$ where $k \geq i$ and $l \leq j$ (or $k \leq i$ and $l \geq j$ ) is impossible, so must be forbidden by the program. The constraints can be written as:

$$
M_{j}^{i}+M_{l_{1}}^{k_{1}} \leq 1, M_{j}^{i}+M_{l_{2}}^{k_{2}} \leq 1, \ldots, M_{j}^{i}+M_{l_{t_{i}}}^{k_{t_{i}}} \leq 1
$$

where $t_{i}$ is the number of matches conflicting with $M_{j}^{i}$, and for any $M_{l_{u}}^{k_{u}}$ we have either $k_{u} \geq i$ and $l_{u} \leq j$, or $k_{u} \leq i$ and $l_{u} \geq j$. There are at most a linear number of inequalities for each of the possible $O\left(n^{2}\right)$ matches.

Equality 1 ensures that each character will be covered by exactly one $D, M$, or $L$. Our objective function minimizes some linear combination of all $L \mathrm{~s}$ and all $D \mathrm{~s}$ :

$$
\min c_{1} L X^{1}+\cdots+c_{n} L X^{n}+c_{n+1} L Y^{1}+\cdots+c_{n+m} L Y^{m}+c_{n+m+1} D_{1}+\cdots+c_{n+m+q} D_{q}
$$

where $c_{l}$ is a cost of the $l$ th operation and $q$ is the total number of duplications for all positions of $X$ and $Y$ (i.e. $D_{l}=D X_{s}^{i}$ or $D_{l}=D Y_{r}^{j}$ for some $i, j, s$, and $r$ ). The full PBLP (excluding the trivial integrality constraints) is then:

$$
\begin{array}{lll}
\min & c_{1} L X^{1}+\cdots+c_{n} L X^{n}+c_{n+1} L Y^{1}+\cdots+c_{n+m} L Y^{m}+ & \\
& c_{n+m+1} D_{1}+c_{n+m+2} D_{2}+\cdots+c_{n+m+q} D_{q} \\
\text { s.t. } & L X^{i}+M_{1}^{i}+M_{2}^{i}+\cdots+M_{p_{i}}^{i}+D X_{1}^{i}+\cdots+D X_{s_{i}}^{i}=1, \quad 0 \leq i \leq n \\
& L Y^{j}+M_{j}^{1}+M_{j}^{2}+\cdots+M_{j}^{q_{j}}+D Y_{1}^{j}+\cdots+D Y_{r_{j}}^{j}=1, \quad 0 \leq j \leq m \\
& M_{j}^{i}+M_{l_{1}}^{k_{1}} \leq 1, M_{j}^{i}+M_{l_{2}}^{k_{2}} \leq 1, \ldots, M_{j}^{i}+M_{l_{t_{i}}}^{k_{t_{i}}} \leq 1 \quad, \quad \forall i, j \text { s.t. } X_{i}=Y_{j} \text { and } \\
& \left(k_{m} \geq i \text { and } l_{m} \leq j\right), \text { or } \\
& \left(k_{m} \leq i \text { and } l_{m} \geq j\right)
\end{array}
$$

In the example illustrated in Figure 2 - where $X=$ "zxyzxyaxbwaxb" and $Y=$ "zxyxwb" - there are 17 variables corresponding to matches, 20 variables corresponding to losses, and 24 variables corresponding to duplications. 
Cyclic Duplications Recall the definition of the product of a duplication; in Figure 2b, the product of the leftmost duplication is $z_{4}, x_{5}$, and $y_{6}$. Consider a sequence of duplications $D_{1}, D_{2}, \ldots, D_{l}$ and characters $a_{1}, a_{2}, \ldots, a_{l}$ such that character $a_{i}$ is in the product of $D_{i}$ and is the duplication of the character $a_{i-1}\left(a_{1}\right.$ is the duplication of some character $\left.a_{0}\right)$. We call this set of duplications cyclic if $D_{1}=D_{l}$. Consider the set of duplications $\left\{D_{1}, D_{2}\right\}$ where $D_{1}$ duplicates the substring $X_{1} X_{2}$ to produce the substring $X_{3} X_{4}$ and $D_{2}$ duplicates the substring $X_{4} X_{5}$ to produce the substring $X_{1} X_{2}$. This implies the sequence of characters $X_{2}, X_{4}, X_{1}, X_{3}$ corresponding to the cyclic duplication $D_{1}, D_{2}, D_{1}$.

Theorem 2. A solution to the PBLP of Section 4.1 that has no cyclic set of duplications is an optimal solution to the Duplication-Loss Alignment problem.

Proof. Equation 1 ensures that each character of $X$ is either aligned to a character of $Y$, aligned to a loss in $Y$, or the product of a duplication. The similar holds for each character of $Y$. Since there exists no cyclic set of duplications, then the solution given by the PBLP is a feasible solution to the Duplication-Loss Alignment problem. The minimization of Formula 3 guarantees optimality.

However, if there does exist a cyclic duplication set, the solution given by the PBLP is not a feasible solution since the cycle implies a scenario that is impossible; the cycle implies a character that does not exist in the ancestor but does appear in $X$. A cyclic duplication set $\left\{D_{1}, D_{2}, \ldots, D_{l}\right\}$ can be forbidden from a solution of the PBLP by the inclusion of the following inequality:

$$
D_{1}+D_{2}+\cdots+D_{l} \leq l-1 \text {. }
$$

The following algorithm guarantees an acyclic solution to the PBLP. It simply runs the PBLP

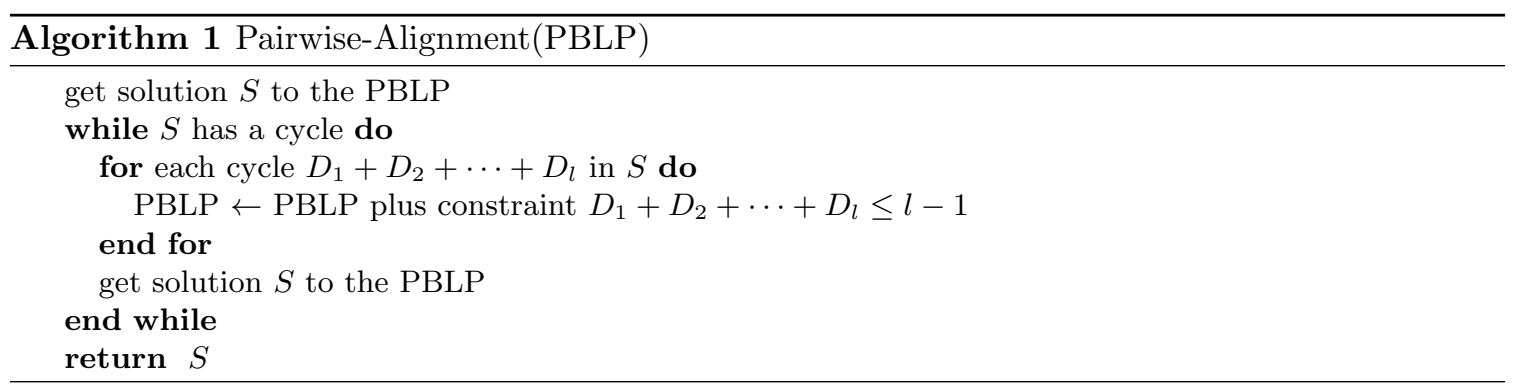

and each time it finds a cyclic set of duplications, it adds the corresponding constraint to forbid the set and reruns the PBLP. It is clear that the algorithm of Figure 1 guarantees an acyclic solution:

Theorem 3. Algorithm 1 returns an optimal solution to the Duplication-Loss Alignment problem.

Note that the constraints to forbid all possible cyclic sets of duplications, given a particular $X$ and $Y$, could be added to the PBLP from the start, but in the worst case there is an exponential number of such constraints. We will see in Section 5.2 that in practice we do not have to rerun the PBLP many times to find an acyclic solution.

\section{Applications}

\subsection{Evolution of stable RNA gene content and organization in Bacillus}

The stable RNAs are chiefly transfer RNAs (tRNAs) and ribosomal RNAs (rRNAs), which are essential in the process of translating messenger RNAs (mRNAs) into protein sequences. They 
are usually grouped in the genome within clusters (or operons in the case of microbial genomes), representing highly repetitive regions, causing genomic instability through illegitimate homologous recombination. In consequence, stable RNA families are rapidly evolving by duplication and loss [35, $3,28]$.

We applied Algorithm 1 in a phylogenetic context, using the SPP-HEURISTIC described at the end of Section 2, to analyze the stable RNA content and organization of 5 Bacillus lineages: Bacillus cereus ATCC 14579 (NC4722), Bacillus cereus E33L (NC6274), Bacillus anthracis (NC7530), Bacillus licheniformis ATCC 14580 (NC6322) and Bacillus subtilis (NC964). The overall number of represented RNA families in these genomes is around 40, and the total number of RNAs in each genome is around 120. Our PBLP algorithm processes each pair of these genomes in a few seconds. We used the following cost for duplications and losses: $c(D(k))=1$ and $c(L(k))=k$, for any integer $k$ representing the size of an operation. The phylogeny in Figure 3 reflects the NCBI taxonomy. Each leaf is labeled by a block representation of the corresponding genome. Details on each colored block is given in Figure 4 of the appendix.

The costs and evolutionary scenarios given in Figure 3 are those output by our algorithm after interpretation. In particular, the five Bacillus genomes all show a large inverted segment in the region to the left of the origin of replication (the right part of each linearized representation in Figure 3). As our algorithm does not handle inversions, we preprocessed the genomes by inverting this segment. The genome representations given in Figure 3 are, however, the true ones. Signs given below the red bars represent their true orientations. Consequently, the duplication of the right-most red bar to the left-most position should be interpreted as an inverted block duplication that occurred around the origin of replication. On the other hand, some duplications of the red bar have been reported by our algorithm as two separate duplications of two segments separated by a single gene. After careful consideration (see Figure 5 of the appendix), these pairs of duplications are more likely a single duplication obscured by subsequent substitution, functional shift or loss of a single tRNA gene. Also, when appropriate (e.g. when a lone gene is positioned in a lone genome), we interpreted some of the losses in our alignments as insertions.

The ancestral genomes given in Figure 3 are those output by our algorithm. They reflect two separate inverted duplications that would have occurred independently in each of the two groups (cereus, anthracis) and (licheniformis, subtilis). We could alternatively infer that the ancestral Bacillus genome already contained both the origin and product of the inverted duplication. The consequence would be the simultaneous loss of the leftmost red bar in three of the five considered genomes. Moreover, nucleotide sequence alignment of the red bars in subtilis and cereus ATCC reveal a higher conservation of pairs of paralogous bars versus orthologous ones, which may indicate that inverted duplications are recent. Whatever the situation is, our inference of a duplication around the origin of replication is in agreement with the observation that has been largely reported in the literature that bacterial genomes have a tendency to preserve a symmetry around the replication origin and terminus $[13,33,1]$. The results also show independent proliferation of ribosomal RNA gene-containing operons in Bacillus, which has been associated to selection for increased growth rate [2]. They also show that in Bacillus, growth-selection on ribosomal RNA operon expansions may significantly alter tRNA gene content as well. The results given in Figure 5 of the appendix also suggest that some tRNA genes may have been affected by substitutions leading to conversions of function. Such tRNA functional shifts have been detected in metazoan mitochondrial genomes [27] and bacteria [29].

\subsection{Execution time}

Running times were recorded using a 12-core AMD 2.1GHZ processor, with 256GB of RAM, and the (multithreaded) IBM CPLEX solver under the default settings. Note that a significant amount of memory $(>1 \mathrm{~GB})$ was required only for sequences of several thousand genes; all tests reported here could be run on a standard laptop with 2 GB of memory. Alignments of all pairs of genomes 


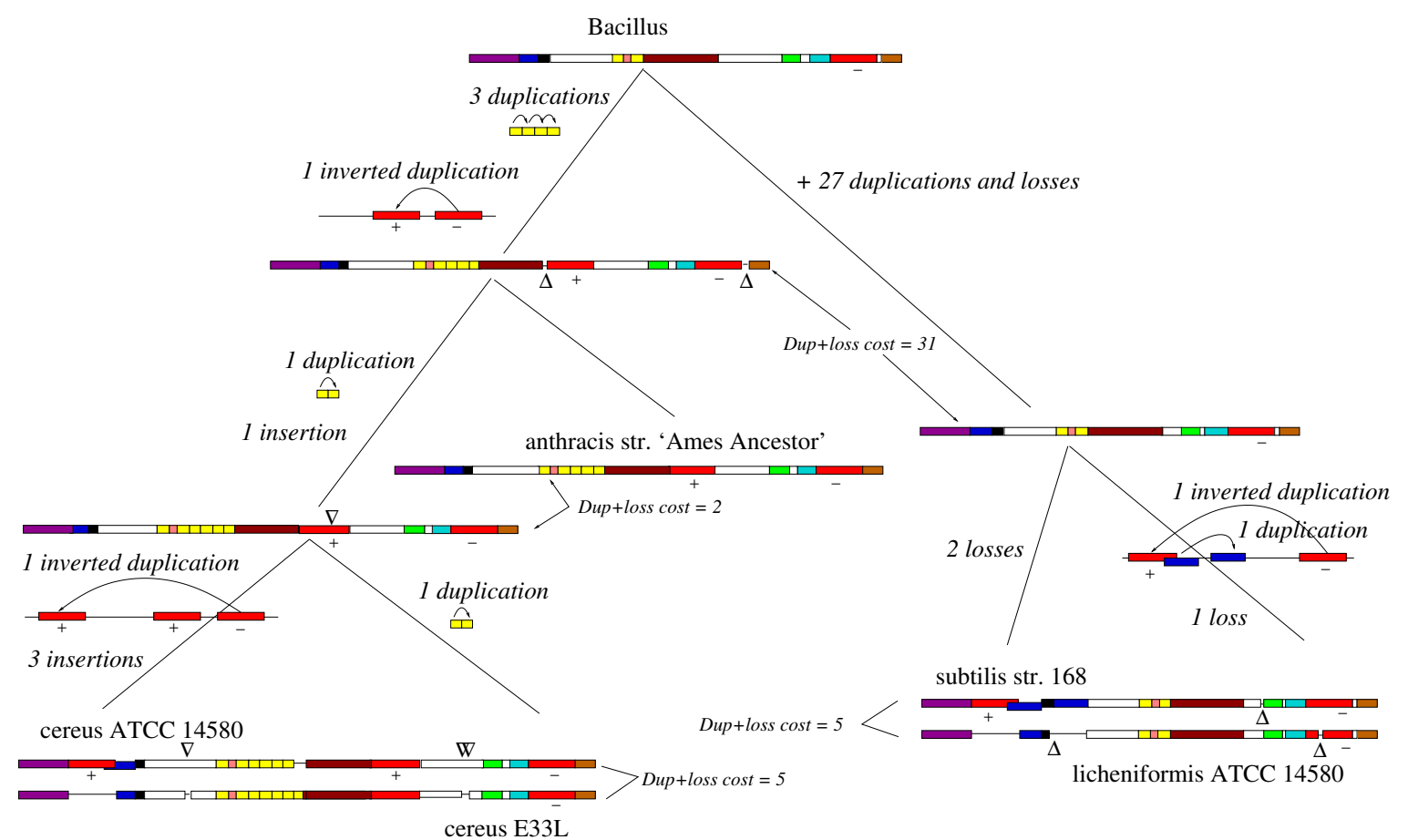

Fig. 3: An inferred evolutionary history for the five Bacillus lineages identified with each of the five leaves of the tree. Circular bacterial genomes have been linearized according to their origin of replication (e.g. the endpoints of each genome is its origin of replication). Bar length is proportional to the number of genes in the corresponding cluster. A key for the bars is given in Figure 4, except for white bars that represent regions that are perfectly aligned inside the two groups (cereus, anthracis) and (licheniformis, subtilis), but not between the two groups. More specifically, the 27 duplications and losses reported on the top of the tree are obtained from the alignment of these white regions. Finally, each $\Delta$ represents a loss and each $\nabla$ is an insertion.

for each of three sets of stable RNA gene orders were computed. The average computation time for the Bacillus pairs was under thirty seconds. The average computation time for pairs from 13 Staphylococcus was under a second. Pairs from a dataset of Vibrionaceae which had a very high number of paralogs and a large number of rearrangements took a couple of days.

\subsection{Simulations}

Simulations were run in order to explore the limits of our method (full results not shown due to space limitations). A random sequence $R$ was drawn from the set of all sequences of length $n$ and alphabet size $a . l$ moves were then applied to $R$ to obtain the ancestral sequence $A$. To obtain the extant sequences $X$ and $Y, l$ more moves were applied to $A$ for each. The set of moves were segmental duplications and single gene losses. The length of a duplication was drawn from a Gaussian distribution with mean 5 and standard deviation 2; these lengths were consistent with observations on Bacillus and Staphylococcus. Average running times for sequences with a fixed ratio of $2 l / n=1 / 5$ and $a / n=1 / 2$ (statistics similar to those observed in Bacillus) were always below 6 minutes for $n<800$. Sequences of length 2000 took less than 2 hours and sequences of length 5000 took a couple of days. When varying $l, n$, and $a$ the most telling factor for running time was the ratio $a / n$. This explains the high running times for the set of Vibrionaceae which had, on average, nearly 100 moves for a sequence of length 140. 
The distance of our computed ancestor to the simulated ancestor was found by computing an alignment between the two. For values of $n=120, a=40$, and $l=15$ (values that mimic the statistics of more distant pairs of the Bacillus data) we compute ancestors that are, on average, 5 moves away from the true ancestor. In general, for sequences with ratios $2 l / n=1 / 5$ and $a / n=1 / 2$, the average distance to the true ancestor stays at about $15 \%$ of $l$.

\section{Conclusion}

We have considered the two species small phylogeny problem for an evolutionary model reduced to content-modifying operations. Although exponential in the worst case, our pseudo-boolean linear programming algorithm turns out to be fast on real datasets, such as the RNA gene repertoire of bacterial genomes. We have also explored avenues for developing efficient non-optimal heuristics. As described in Section 4, a dynamic programming approach can be used to infer a reasonable, though not necessarily optimal unlabeled alignment of two genomes. An important open problem is then to discern the complexity of finding a minimum labeling for a given alignment. The implication being that if this problem is NP-hard, then the two species small phylogeny problem is not even in NP.

Application to the Bacillus lineages has pointed out a number of generalizations that could be introduced to the evolutionary model, such as inversions, inverted duplications, gene conversion (substitutions), and insertions. Substitutions can easily be accommodated in our PBLP by allowing matching of different genes, and attributing a cost according to the likelihood of that gene conversion. Our boolean programming model is also likely to handle "visible", in term of non-crossing inversions and inverted duplications. As for insertions, they can not be distinguished from losses by the comparison of pairs of genomes. A deterministic methodology could, however, be established to distinguish between them based on results obtained on a complete phylogenetic tree.

\section{References}

1. Y. Ajana, J.F. Lefebvre, E. Tillier, and N. El-Mabrouk. Exploring the set of all minimal sequences of reversals - an application to test the replication-directed reversal hypothesis. In LNCS, volume 2452 of $W A B I$, pages 300-315, 2002.

2. D.H. Ardell and L.A. Kirsebom. The genomic pattern of tDNA operon expression in E. coli. PLoS Comp. Biol., 1(1:e12), 2005.

3. C. Bermudez-Santana, C. S. Attolini, T. Kirsten, J. Engelhardt, S.J. Prohaska, S. Steigele, and P. Stadler. Genomic organization of eukaryotic tRNAs. BMC Genomics, 11(270), 2010.

4. M. Blanchette, G. Bourque, and D. Sankoff. Breakpoint phylogenies. Genome Informatics Workshop (GIW), pages 25- 34, 1997.

5. T. Blomme, K. Vandepoele, S. De Bodt, C. Silmillion, S. Maere, and Y. van de Peer. The gain and loss of genes during 600 millions years of vertebrate evolution. Genome Biology, 7:R43, 2006.

6. G. Bourque and P.A. Pevzner. Genome-scale evolution: Reconstructing gene orders in the ancestral species. Genome Research, 12:26 - 36, 2002.

7. A. Caprara. Formulations and hardness of multiple sorting by reversals. In RECOMB, pages 84-94, 1999.

8. C. Chauve and E. Tannier. A methodological framework for the reconstruction of contiguous regions of ancestral genomes and its application to mammalian genomes. PloS Computational Biology, 4:e1000234, 2008.

9. J.A. Cotton and R.D.M. Page. Rates and patterns of gene duplication and loss in the human genome. Proceedings of the Royal Society of London. Series B, 272:277-283, 2005.

10. J.P. Demuth, T. De Bie, J. Stajich, N. Cristianini, and M.W. Hahn. The evolution of mammalian gene families. PLoS ONE, 1:e85, 2006.

11. H. Dong, L. Nilsson, and C. G. Kurland. Co-variation of tRNA abundance and codon usage in Escherichia coli at different growth rates. Journal of Molecular Biology, 260:649-663, 2006. 
12. E.E. Eichler and D. Sankoff. Structural dynamics of eukaryotic chromosome evolution. Science, 301:793$797,2003$.

13. J.A. Eisen, J.F. Heidelberg, O. White, and S.L. Salzberg. Evidence for symmetric chromosomal inversions around the replication origin in bacteria. Genome Bioloy, 1(6), 2000.

14. N. El-Mabrouk. Mathematics of Evolution and Phylogeny, chapter Genome rearrangement with gene families, pages 291- 320. Oxford University Press, 2005.

15. G. Fertin, A. Labarre, I. Rusu, E. Tannier, and S. Vialette. Combinatorics of genome rearrangements. The MIT Press, Cambridge, Massachusetts and London, England, 2009.

16. M.W. Hahn, M.V. Han, and S.-G. Han. Gene family evolution across 12 drosophilia genomes. PLoS Genetics, 3:e197, 2007.

17. S. Hannenhalli and P. A. Pevzner. Transforming men into mice (polynomial algorithm for genomic distance problem). In Proceedings of the IEEE 36th Annual Symposium on Foundations of Computer Science, pages 581-592, 1995.

18. S. Hannenhalli and P. A. Pevzner. Transforming cabbage into turnip (polynomial algorithm for sorting signed permutations by reversals). Journal of the ACM, 48:1-27, 1999.

19. Minghui Jiang. The zero exemplar distance problem. In RECOMB-CG, pages 74-82, 2010.

20. S. Kanaya, Y. Yamada, Y. Kudo, and T. Ikemura. Studies of codon usage and tRNA genes of 18 unicellular organisms and quantification of Bacillus subtilis tRNAs: Gene expression level and speciesspecific diversity of codon usage based on multivariate analysis. Gene, 238:143-155, 1999.

21. J. Kovac, B. Brejova, and T. Vinar. A practical algorithm for ancestral rearrangement reconstruction. In $L N B I$, volume 6833 of $W A B I$, pages 163- 174, 2011.

22. M. Lynch and J.S. Conery. The evolutionary fate and consequences of duplicate genes. Science, 290:1151$1155,2000$.

23. J. Ma, L. Zhang, B.B. Suh, B.J. Raney, R.C. Burhans, W.J. Kent, M. Blanchette, D. Haussler, and W. Miller. Reconstructing contiguous regions of an ancestral genome. Genome Research, 16:1557 1565, 2007.

24. B. Moret, L. Wang, T. Warnow, and S. Wyman. New approaches for reconstructing phylogenies from gene order data. Bioinformatics, 17:S165-S173, 2001.

25. S. Ohno. Evolution by gene duplication. Springer, Berlin, 1970.

26. I. Pe'er and R. Shamir. The median problems for breakpoints are NP-complete. Elec. Colloq. on Comput. Complexity, 71, 1998.

27. T.A. Rawlings, T.M. Collins, and R. Bieler. Changing identities: tRNA duplication and remolding within animal mitochondrial genomes. Proceedings of the National Academy of Sciences USA, 100:15700-15705, 2003.

28. H.H. Rogers, C.M. Bergman, and S. Griffiths-Jones. The evolution of tRNA genes in Drosophila. Genome Biol. Evol, 2:467- 477, 2010.

29. M.E. Saks and J.S. Conery. Anticodon-dependent conservation of bacterial tRNA gene sequences. $R N A$, 13(5):651- 660, 2007.

30. D. Sankoff and M. Blanchette. The median problem for breakpoints in comparative genomics. In T. Jiang and D.T. Lee, editors, Computing and Combinatorics, Proceeedings of COCOON '97, number 1276 in Lecture Notes in Computer Science, pages 251-263, Berlin, 1997. Springer.

31. D.T. Tang, E.A. Glazov, S.M. McWilliam, W.C. Barris, and B.P. Dalrymple. Analysis of the complement and molecular evolution of tRNA genes in cow. BMC Genomics, 10(188), 2009.

32. E. Tannier, C. Zheng, and D. Sankoff. Multichromosomal median and halving problems under different genomic distances. BMC Bioinformatics, 10, 2009.

33. E.R.M. Tillier and R.A. Collins. Genome rearrangement by replication-directed translocation. Nature Genetics, 26, 2000.

34. I. Wapinski, A. Pfeffer, N. Friedman, and A. Regev. Natural history and evolutionary principles of gene duplication in fungi. Nature, 449:54-61, 2007.

35. M. Withers, L. Wernisch, and M. Dos Reis. Archaeology and evolution of transfer RNA genes in the escherichia coli genome. Bioinformatics, 12:933-942, 2006. 


\section{A Alignment Details}

Ribosomal RNAs are grouped in bacteria into three families: the $16 \mathrm{~S}, 5 \mathrm{~S}$ and $23 \mathrm{~S}$ rRNAs. As the major role of tRNAs is to serve as adapters between codons along the mRNA and the corresponding amino acids, we group them according to their anticodon. More precisely, the four-letter designation starts with one letter indicating functional class (either an IUPAC one-letter code for a charged amino acid, "X" for initiator or "J" for a special class of isoleucine tRNA) followed by an anticodon sequence in a DNA alphabet. The full alignment as given by our program can be retrieved at: http://lcbb4.epfl.ch/web/BacillusAlignements/.

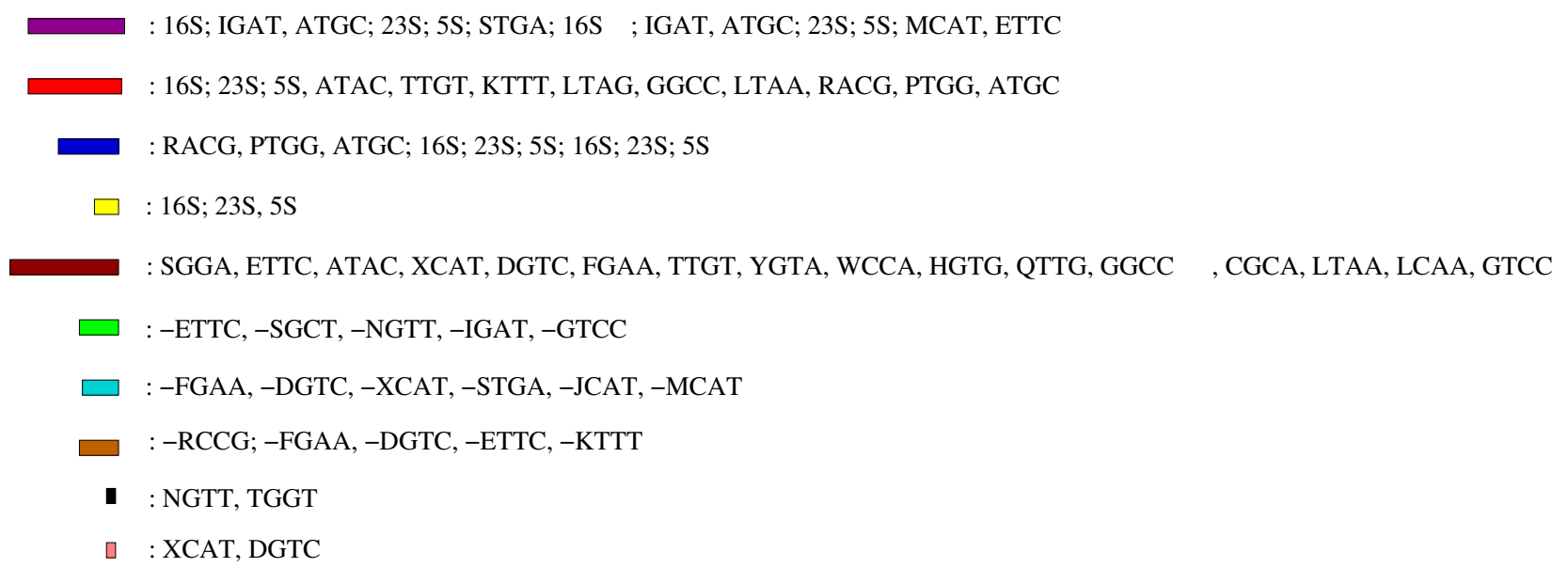

Fig. 4: The RNA gene clusters represented by each colored bar of Figure 3. Ribosomal RNAs are identified by their families' names: $16 \mathrm{~S}, 23 \mathrm{~S}, 5 \mathrm{~S}$. Each tRNA is identified by its anticodon preceded by the letter corresponding to the corresponding amino-acid. The symbol ',' (respectively ';') separates two genes that are inside (resp. not inside) the same operon.

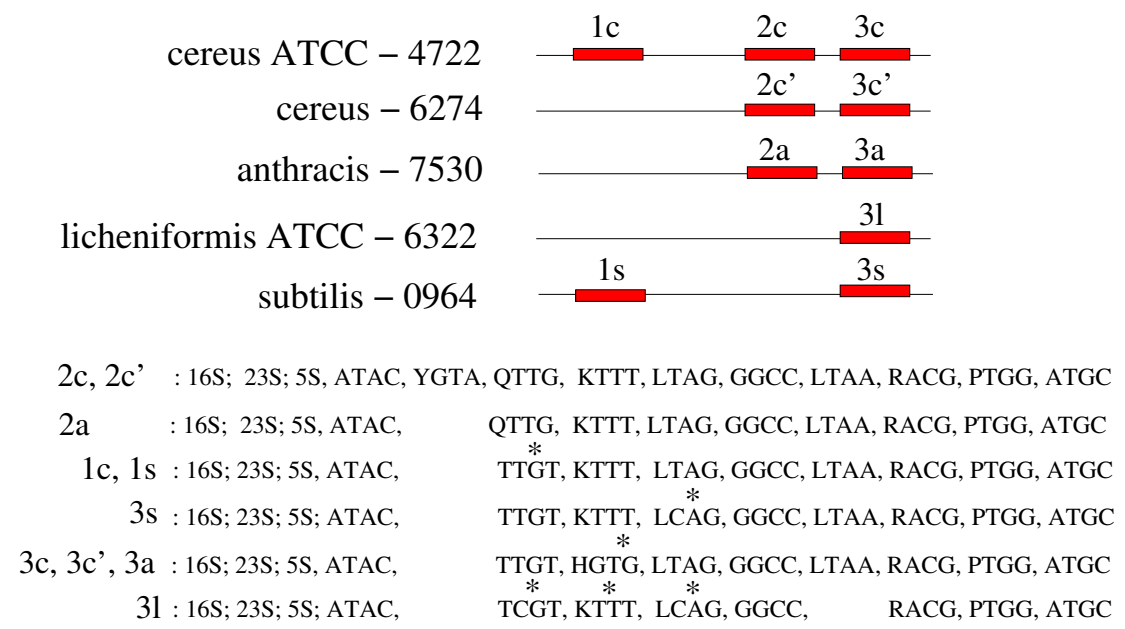

Fig. 5: (a) Genomes of Figure 3 restricted to their red bars; (b) An alignment of all red bars, reflecting one gene insertion (or alternatively 5 gene losses) and substitutions, indicated by '*'. 


\section{B Proof of Theorem 1}

Proof. Let $A$ be a visible ancestor of $X$ and $Y$ and $\left(A, O_{A \rightarrow X}, O_{A \rightarrow Y}\right)$ be a visible history of $X$ and $Y$. Then construct a labeled alignment $\mathcal{A}$ of $X$ and $Y$ as follows:

1. Initialization: Define the two strings $X^{-}=Y^{-}=A$ on $\Sigma^{-}$, and define $\mathcal{A}$ as an alignment with all matches between $X^{-}$and $Y^{-}$(i.e. self-alignment of $A$ ).

2. Consider each operation of $O_{A \rightarrow X}$ in order.

- If it is a duplication, then add the inserted string at the appropriate position in $X^{-}$, and add gaps (" - " characters) at the corresponding positions in $Y^{-}$. Label the inserted columns of $\mathcal{A}$ as a duplication in $X$, coming from the columns of the alignment representing the origin of the duplication.

- If it is a loss, then replace the lost characters in $X^{-}$by gaps. Label the modified columns as a loss in $X$.

3. Consider each operation of $O_{A \rightarrow Y}$ and proceed in a symmetrical way.

As $\left(A, O_{A \rightarrow X}, O_{A \rightarrow Y}\right)$ is a visible history of $X$ and $Y$, by definition the origins and products of duplications remain unchanged by subsequent operations on each of $O_{A \rightarrow X}$ and $O_{A \rightarrow Y}$. Therefore, all intermediate labellings remain valid in the final alignment. Therefore, the constructive method described above leads to a labeled alignment of $X$ and $Y$.

On the other hand, a substring $X_{i} \cdots X_{j}\left(\right.$ resp. $Y_{i} \cdots Y_{j}$ ) that is labeled as a duplication in $X$ (resp. $Y$ ) should not be present in $A$, as it is duplicated on the branch from $A$ to $X$ (resp. from $A$ to $Y$ ). Also, a substring $X_{i} \cdots X_{j}$ (resp. $Y_{i} \cdots Y_{j}$ ) that is labeled as a loss in $Y$ (resp. $X$ ) should be present in $A$, as it is lost on the branch from $A$ to $Y$ (resp. from $A$ to $X$ ). This implies an obvious algorithm to reconstruct a unique ancestor. 